\title{
Testing Adaptations of Cognitive-Behavioral Conjoint Therapy for PTSD: A Randomized Controlled Pilot Study With Veterans
}

\author{
Louanne W. Davis ${ }^{1,2}$, Brandi L. Luedtke ${ }^{3}$, Candice Monson ${ }^{4}$, Alysia Siegel $^{5}$, \\ Joanne K. Daggy ${ }^{6}$, Ziyi Yang ${ }^{6}$, Matthew J. Bair ${ }^{7,8,9}$, \\ Beth Brustuen ${ }^{10}$, and Michelle Ertl ${ }^{11}$ \\ ${ }^{1}$ Psychiatry Department, Roudebush VA Medical Center, Indianapolis, Indiana, United States \\ ${ }^{2}$ Department of Psychiatry, Indiana University School of Medicine \\ ${ }^{3}$ Department of Psychology, Carl T. Hayden VA Medical Center, Phoenix, Arizona, United States \\ ${ }^{4}$ Department of Psychology, Ryerson University \\ ${ }^{5}$ Trauma Recovery Program, James A. Haley Veterans' Hospital, Tampa, Florida, United States \\ ${ }^{6}$ Department of Biostatistics, Indiana University School of Medicine \\ ${ }^{7}$ Center for Health Information and Communication, Roudebush VA Medical Center, Indianapolis, \\ Indiana, United States \\ ${ }^{8}$ Department of Internal Medicine, Indiana University School of Medicine \\ ${ }^{9}$ Department of Internal Medicine, Regenstrief Institute, Indianapolis, Indiana, United States \\ ${ }^{10}$ School of Psychological Sciences, University of Indianapolis \\ ${ }^{11}$ Trauma, Stress, and Resilience Program, Utah Center for Evidence Based Treatment, Salt Lake City, \\ Utah, United States
}

\begin{abstract}
Iraq and Afghanistan Veterans with posttraumatic stress disorder (PTSD) have welldocumented relationship problems and many wish to include their intimate partners in treatment. This pilot study randomly assigned 46 couples (Veterans with clinicianadministered PTSD scale confirmed PTSD diagnosis and their intimate partners) to one of two groups. The treatment group received a modified mindfulness-based version of cognitive-behavioral conjoint therapy for PTSD (CBCT; Monson \& Fredman, 2012) that included all three phases of the mindfulness-based cognitive behavioral conjoint therapy (MB-CBCT). The control group received a modified version of CBCT that included communication skills training from Phases 1 and 2 of CBCT (CBCT-CS) without PTSDspecific content. Modified CBCT Phases 1 and 2 content was delivered to both groups
\end{abstract}

Louanne W. Davis (D) https://orcid.org/0000-0002-1485$124 \mathrm{X}$ 0788

Candice Monson (iD https://orcid.org/0000-0001-6179-

Alysia Siegel (D) https://orcid.org/0000-0002-0907-0449

Michelle Ertl (D) https://orcid.org/0000-0002-2508-7443

This research was supported by grants from Veterans Administration Rehabilitation Research and Development. Views expressed in this article are those of the authors and do not necessarily represent the views of the Department of Veterans Affairs or the U.S. government. We thank Amanda Kovach and Corrie Stewart for providing study coordination; Alexandra McDonald for training and consultation with study assessors; Linda Brown for delivering CBCT-CS with assistance from Jena Wierwille, Rachel Niemi, and Amanda Kovach;
Sarah Mallis, Zachary Michaels, Scott Bischoff, Erica Birkley, Kyle Olesek, Jonathan Lassiter and Corrie Stewart for assisting Louanne W. Davis and Brandi L. Luedtke with delivery of CBCT interventions; Travis Slesinski, Jaclyn Hillis, Beth Brustuen, Alysia Seigel, and Michelle Ertl for assisting with study assessments during the RCT; Madeline Brennan and Megan Mischler for assisting with study assessments during the manual development phase; Licci Jaimes, Marissa Ohlstrom, Matthew Lessack and Shelby Casper for assistance with data entry and Barbara Niles, Diana Kamila, and Pat Sheehan for their assistance with the adaptation CBCT for this study. We also thank all the study participants for their valuable contributions.

Correspondence concerning this article should be addressed to Louanne W. Davis, Psychiatry Department, Roudebush VA Medical Center, 724 Greenford Trail North, Carmel, Indiana 46032, United States. Email: 1wdavis@iu.edu 


\begin{abstract}
during weekend retreats in multicouple group sessions. The postretreat protocol for MB-CBCT included nine individual couple sessions: a transition session following the retreat, and CBCT Phase 3. For CBCT-CS, two additional monthly multicouple group sessions reviewed communication skills. No statistically significant pre- to posttreatment differences were detected for primary outcomes between groups: Clinician-Administered PTSD Scale for Veterans (mean change difference, $-1.4,95 \%$ CI [ -16.0 to 13.2]); Dyadic Adjustment Scale for Veterans (mean change difference, $-1.0,95 \%$ CI [ -13.2 to 11.2]); and Dyadic Adjustment Scale for Partners (mean change difference, $-0.4,95 \%$ CI [ -8.9 to 8.1]). However, within group pre- to posttreatment effect sizes were medium to large for both MB-CBCT and CBCT-CS on all three primary outcomes. Findings suggest that Veterans returning from recent conflicts and their partners may benefit from both modifications of CBCT.
\end{abstract}

Keywords: couple therapy, PTSD, CBCT, cognitive-behavioral therapy, mindfulness

Supplemental materials: https://doi.org/10.1037/cfp0000148.supp

An estimated 10\%-20\% of Veterans who served in Iraq and Afghanistan are diagnosed with posttraumatic stress disorder (Hoge, 2011). Research findings have shown that intimate relationship conflict is associated with Veterans' posttraumatic stress disorder (PTSD) symptoms (Taft et al., 2011). Most available PTSD treatments do not target relationship conflict, which is unfortunate as couple/family functioning moderates responses to individual, evidence-based treatments for PTSD (Dorrington et al., 2018; Meis et al, 2010). Further, many individuals with PTSD wish to include their intimate partners in treatment (Monson et al., 2018). Clinicians working with Veterans who served in Iraq and Afghanistan at the VA Medical Center in Indianapolis also identified a need for programming that includes partners in treatment and addresses both relationship issues and PTSD. As a result, clinicians developed and conducted weekend postdeployment reunification retreats called Operation Restoration (see Davis et al., 2012).

Beginning in 2008, Operation Restoration retreats were held at a retreat center and included sessions on basic communication skills and mindfulness, as well as other topics. The majority of Veterans who attended were diagnosed with PTSD. Program evaluations conducted at the end of the retreats inquired about recommended formats and topics for future programming to address postdeployment issues. Couples indicated their interest in more weekend retreat programming to help them deal with PTSD and relationship issues as well as to build mindfulness skills. Therefore, we envisioned developing and offering another program that was mindfulness-based and addressed both PTSD and relationship functioning utilizing a weekend retreat format. This method of developing clinical programming is in line with recent Veterans Health Administration and the Office of Mental Health and Suicide Prevention mandates for Veteran-centric, approaches to wellness (https://www.va.gov/ wholehealth). As a first step, we searched for existing programs that engaged couples in addressing PTSD and relationship issues.

\section{Conjoint Therapy Addressing PTSD and the Relationship}

Our search for existing programs revealed one program that closely matched the interests of Veterans and their intimate partners at our VA Medical Center: cognitive-behavioral conjoint therapy for PTSD (CBCT for PTSD; Monson \& Fredman, 2012). CBCT for PTSD is a 15session protocol divided into three phases. Phase 1 consists of two sessions. The first session outlines the rationale for treatment and provides education about PTSD and relationships while also helping couples identify treatment goals and increase positivity. The second session focuses on building safety within the relationship by addressing negative behaviors such as anger and managing conflict with time-out. Phase 2 (Sessions 3-7) provides education and skill development designed to increase relationship satisfaction (i.e., reflective listening, sharing thoughts and feelings, problem-solving) in addition to reducing avoidance through in-vivo approach assignments and a dyadic approach to challenging cognitions that maintain PTSD and 
relationship problems. Phase 3 (Sessions 8-15) facilitates making meaning of the trauma by capitalizing on couples' improved communication skills and their shift to approaching rather than avoiding trauma-related issues. During Sessions $8-14$, couples use a dyadic approach to challenging trauma-related cognitions related to acceptance, blame, trust, control, emotional closeness, physical closeness, and posttraumatic growth. Session 15 reviews and reinforces treatment gains and helps couples plan for future challenges.

Based on promising evidence of efficacy, we decided to use CBCT for PTSD as the framework for our new program for Veterans and their intimate partners at our medical center. A recent review of 14 CBCT studies found CBCT to be efficacious for improving PTSD symptoms and individuals with higher relationship distress at baseline had greater improvements in relationship satisfaction (Liebman et al., 2020). We presumed that the 15 week duration of the CBCT protocol as originally designed might pose a barrier to treatment, particularly for couples juggling family, work, and educational pursuits (Erbes et al., 2009). Therefore, we adapted Phases 1 and 2 of CBCT to be implemented during a multicouple weekend retreat thereby allowing the entire 15 week CBCT protocol to be completed in 10 weeks. Since couples who attended Operation Restoration postdeployment retreats also expressed interest in mindfulness, we also reviewed the literature on mindfulness in the context of intimate relationship and PTSD.

\section{Mindfulness as an Intervention for PTSD}

Mindfulness has been described as the ability to remain focused on the reality of the present moment, accepting and being open to it, without getting caught up in elaboration of thoughts or emotional reactions to situations (Kabat-Zinn, 1990). A review of 12 mindfulness-based intervention studies published from 2010 to 2014 that used a reliable, validated measure of PTSD preand postintervention showed PTSD symptom reduction, particularly related to avoidance (Banks et al., 2015). PTSD is currently defined by core symptoms of intrusion (e.g., unwanted memories or flashbacks), avoidance of trauma reminders (e.g., internal or external stimuli that are linked with trauma memories), negative alterations in cognitions and mood (e.g., beliefs about the self, world, and others that drive painful emotions) and alterations in arousal (e.g., hypervigilance, irritability; American Psychiatric Association, 2013). Results of these studies suggest several mechanisms by which mindfulness exerts an effect on PTSD symptoms. First, attention that is trained to remain in the present moment is the hypothesized mechanism whereby reduced reactivity and increased ability to act with awareness are associated with reduced hyperarousal symptoms. In addition, increased openness to experience and willingness to approach feared stimuli is the hypothesized mechanism for reduced avoidance found in studies of mindfulness-based interventions (e.g., Kimbrough et al., 2010; King et al., 2013). Finally, increased self-compassion appears to drive change in PTSD symptoms (Kearney et al., 2013) and reduce self-blame following mindfulness-based coginitive therapy (King et al., 2013) through the hypothesized mechanism of nonjudgmental acceptance of trauma-related cognitions. Adding further support to these proposed mechanisms by which mindfulness affects PTSD, recent neurobiological findings suggest that mindfulness-based therapies may be effective in restoring top-down modulation of limbic regions with associated increases in modulation of emotional reactivity among individuals with PTSD (Boyd et al., 2018). These benefits of mindfulness interventions can be expected to enhance cognitive therapy that targets the beliefs maintaining PTSD and relationship dysfunction.

\section{Mindfulness and Conjoint Therapy}

There are three theoretical rationales for using mindfulness to enhance couples' relationship functioning/satisfaction, according to Carson et al. (2004). First, as in other meditation techniques, mindfulness meditation is thought to induce the relaxation response which involves psychophysiological changes that are the opposite of those associated with stress-induced hyperarousal. Gottman (1993) suggested that psychophysiological soothing is likely to make possible a calmer approach to the shared difficulties and challenges involved in intimate relationships. Second, mindfulness places a fundamental emphasis on experiencing each moment as it is, suspending judgment and cultivating openness and acceptance, which is associated with increased compassion for self and others 
often reported by mindfulness practitioners (Shapiro et al., 1998). Theorists who are interested in relationship enhancement (Wenzel \& Harvey, 2001) as well as a number of marital therapy researchers (e.g. Christensen \& Jacobson, 2000) endorse the importance of acceptance in relationships. Third, mindfulness is essentially a way of being in all life experiences rather than a coping strategy to be used only in difficult life situations and, as such, could be expected to be continuously available to enhance interpersonal functioning. A mindfulness-based relationship enhancement intervention was evaluated in a randomized waitlist control design with 44 nondistressed couples (Carson et al, 2004). For those couples who received the intervention, significant improvements (i.e. moderate effect sizes) were found across a variety of measures of relationship functioning/quality and psychological well-being. Mindfulness has also been linked to improved communication, empathy, and relationship well-being (Carson et al., 2004; Gambrel \& Keeling, 2010). Based on the literature supporting the usefulness of mindfulness in improving relationships and addressing PTSD symptoms, we integrated education about mindfulness and brief mindfulness practices in our adaptation of CBCT.

\section{Purpose of the Present Study}

The first goal of the present study was to develop the protocol, which is called mindfulness-based cognitive behavioral conjoint therapy for PTSD (MB-CBCT). The second goal of the present study was to evaluate the effect of MB-CBCT on PTSD symptoms and relationship satisfaction of Iraq and Afghanistan Veterans with a PTSD diagnosis confirmed by the Clinician-Administered PTSD Scale (CAPSIV), and their partners, compared to an active control. To our knowledge, there are no studies that have investigated how mindfulness may improve the response to couples CBT that targets PTSD symptoms and/or relationship functioning. As previously described, interventions delivered separately have a positive impact on PTSD symptoms and relationship functioning. We anticipated integrating the two interventions in the proposed study would prove synergistic.

We hypothesized that MB-CBCT would lead to greater improvement at treatment end in the primary outcomes of Veterans' PTSD symptoms assessed by clinician interview (CAPS-IV) and Veterans' and partners' selfreported relationship satisfaction compared to an active control, cognitive behavioral conjoint therapy-communication skills (CBCT-CS), as MB-CBCT offers interventions that specifically target both relationship satisfaction and PTSD symptoms. CBCT-CS is a modified version of CBCT that includes communication skills training from CBCT Phases 1 and 2 without the PTSD-specific content. (See Method section for a more detailed description of MB-CBCT and CBCT-CS). We also predicted that MB-CBCT would result in greater improvement at treatment end in secondary outcomes, including Veteran and partner reports of Veterans' PTSD symptoms, as well as Veterans' and partners' reports of anxiety, depression, and anger. Finally, we were interested in exploring sustained treatment effects at 6 months, since previous CBCT studies assessed outcomes only at 3 months.

\section{Method}

\section{Participants and Procedure}

Forty-six US Veterans returning from Operation Enduring Freedom and Operation Iraqi Freedom who met criteria for PTSD and their intimate partners were recruited from outpatient psychiatry clinics at the Department of Veterans Affairs hospital in Indianapolis. They were enrolled in the study from 2012 to 2014. Inclusion and exclusion criteria were comparable to previous CBCT studies (e.g., Monson et al., 2012). Veterans had to be diagnosed with PTSD, however, couples were excluded if their partner had a current diagnosis of PTSD. Both partners had to be age 18 or older and commit to maintaining the relationship for the duration of treatment. Veterans were required to be stable on psychotropic medication(s), defined as no major change in dose or type of medication in the previous month. Exclusion criteria for both Veterans and partners included severe partner aggression in the past year, imminent suicidality or homicidality, cognitive impairment that precluded understanding of session content, self-harm in the past 6 months, and uncontrolled bipolar or psychotic disorders. History of substance abuse was permitted, with an agreement to limit use that would interfere with treatment. Participants were asked 
to forego initiating couple therapy or another evidence-based, PTSD-specific therapy while participating in the study. Continuing to engage in supportive therapy such as biweekly or monthly sessions with a mental health clinician as is typical in the VA was allowed.

After obtaining approval from the Indiana University IRB and VA Medical Center (VAMC) Research Committee, participants were recruited primarily through the outpatient Psychiatry/Mental Health Service of the VAMC. The primary recruitment strategy was sending letters to patients who had a PTSD or related diagnosis documented in their medical record, followed by a phone call from study staff. Participants were also recruited by clinician or selfreferral at the VAMC and through brochures distributed to community-based clinics such as the Vet Center. Interested couples met with study staff to receive information, and, if willing to participate, signed informed consents and were assessed for inclusion/exclusion criteria. Veterans' PTSD diagnosis was established by a semistructured clinician interview using the Clinician-Administered PTSD Scale (CAPS-IV; Blake et al., 1995). Partner PTSD was an exclusion criterion. Partners were screened for PTSD by inquiring about their history, and if it was positive for trauma, partners completed the PTSD Checklist (PCL-IV), followed by a CAPS interview if the PCL score was at or above the 30-point cut-off suggestive of diagnosis for civilians for PCL-IV.

After couples provided demographic information and completed baseline assessments, they were randomly assigned to $\mathrm{MB}-\mathrm{CBCT}$ or CBCT-CS. A block randomization procedure was used with blocks of four to ensure balanced treatment groups throughout the study. Fortysix couples were randomized and treated in four waves over 2 years. In order to honor participant choice, we offered MB- CBCT to interested participants who were randomly assigned to CBCT-CS once they completed CBCT-CS and follow-up assessments.

Participants were subsequently assessed at midtreatment (after the retreat and before the first postretreat session), posttreatment, and 6 months after completing treatment. All measures were administered at each time point, except the CAPS, which was not administered midtreatment to reduce participant burden. Each participant was compensated $\$ 25$ for the time involved in completing the assessment at each time point. Gas gift cards were given to offset transportation costs for the weekend retreat as well as for subsequent appointments in cases of financial need. See Figure 1 CONSORT diagram for details of participant flow.

We enrolled 46 of the 48 planned couples. Of the 23 couples that initiated treatment in the MBCBCT group, 17 (74\%) completed the intervention, whereas only nine (39\%) of the 23 couples in the CBCT-CS group completed the intervention (see Figure 1). However, 18 CBCT-CS couples (78\%) completed the weekend retreat, which provided the communication skills training and the majority of in-session practice. Baseline demographic and behavioral characteristics of the 46 PTSD-identified Veterans and their partners are reported in Table 1. Most Veterans were male and Caucasian. One same-sex female couple was randomized to MB-CBCT. The CBCT-CS couples were married on average twice as many years as the MB-CBCT group though this was not statistically significant. MB-CBCT and CBCTCS participants did not differ on most variables, except that Veterans in MB-CBCT were more likely to have experienced a traumatic brain injury (TBI) than CBCT-CS Veterans (13/23 vs. $3 / 23, p=.002)$. On the baseline Dyadic Adjustment Scale (DAS), CBCT-CS partners had significantly lower scores than MB-CBCT partners $($ CBCT-CS, mean $=95.7$, in the distressed range <98; MB-CBCT, mean $=107.6$, in the satisfied range; $p=.046)$. Though not significant, this pattern was also observed in the Veterans $(p=.577)$. On average, CBCTCS Veterans were in the distressed range on the DAS at baseline whereas MB-CBCT Veterans were in the satisfied range.

\section{Treatment Group-MB-CBCT}

MB-CBCT is a manualized adaptation of CBCT a three-phase couple treatment designed to improve PTSD symptoms and relationship satisfaction. While working with a total of seven couples in two waves, we developed the retreat format for CBCT Sessions 1-7, developed a transition session postretreat emphasizing mindfulness and the cognitive interventions from CBCT Sessions 6 and 7, and integrated mindfulness throughout the CBCT protocol. Since increased awareness can sometimes trigger PTSD symptoms, we modified standard mindfulness practices and emphasized the importance of participant choice, for example, which mindfulness 
Figure 1

Participant Flow

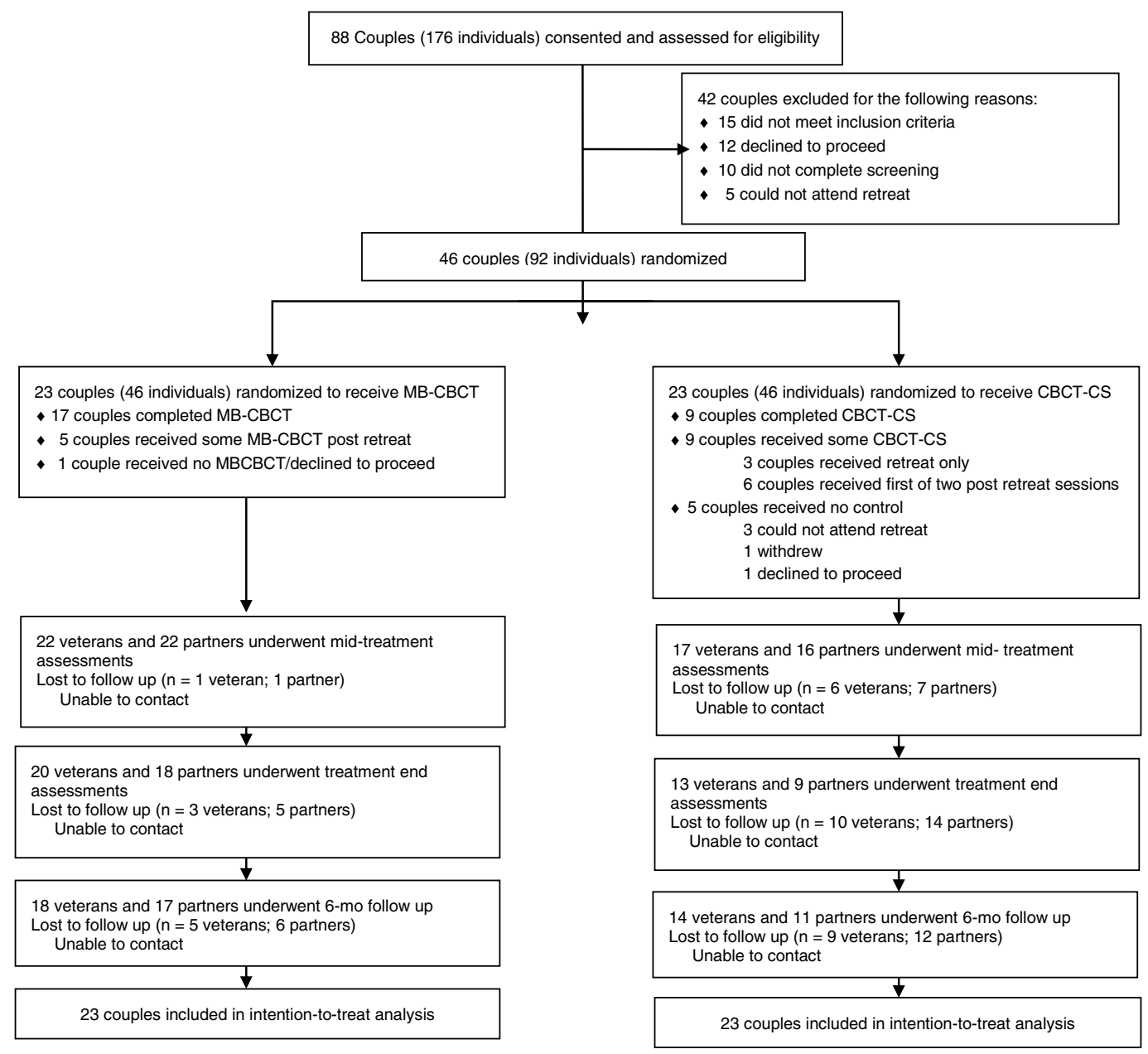

practice to engage in and for how long. Awareness training during the retreat included perception exercises, definition of mindfulness, and supporting research as well as formal and informal skills practice (eating, walking, sitting, driving, and loving-kindness). Postretreat, participants continued practice of awareness skills in and out of sessions. See Davis (2016) for material on mindfulness modifications for individuals who have PTSD. See Luedtke et al. (2015) and Davis et al. (2014) for further detail regarding the $\mathrm{MB}-\mathrm{CBCT}$ protocol.

Three therapists provided MB-CBCT (first and second author and one doctoral-level trainee who conducted postretreat couple sessions independently with one couple). All three MB-CBCT therapists attended a CBCT training workshop with the third author. The first and second author received group consultation with a CBCT consultant trained by the CBCT developers. The first author had been practicing and teaching mindfulness meditation for over 5 years and completed three of the four required trainings for teacher certification in MBSR through the Center for Mindfulness at the University of Massachusetts Medical Center, in addition to participating in yearly silent retreats. The second author had completed two of the four training required for 
Table 1

Baseline Characteristics by Treatment Group and Partner Status

\begin{tabular}{|c|c|c|c|c|c|c|c|c|c|c|}
\hline \multirow[b]{3}{*}{ Baseline characteristics } & \multicolumn{5}{|c|}{ PTSD-identified veterans } & \multicolumn{5}{|c|}{ Partners } \\
\hline & \multicolumn{2}{|c|}{$\begin{array}{c}\text { MB-CBCT } \\
(n=23)\end{array}$} & \multicolumn{2}{|c|}{$\begin{array}{c}\text { CBCT-CS } \\
(n=23)\end{array}$} & \multirow[b]{2}{*}{$p$ value $^{\mathrm{a}}$} & \multicolumn{2}{|c|}{$\begin{array}{c}\text { MB-CBCT } \\
(n=23)\end{array}$} & \multicolumn{2}{|c|}{$\begin{array}{c}\text { CBCT-CS } \\
(n=23)\end{array}$} & \multirow[b]{2}{*}{$p$ value } \\
\hline & $N$ & $\%$ & $N$ & $\%$ & & $N$ & $\%$ & $N$ & $\%$ & \\
\hline Age, years $(M$ and $S D)$ & 38.9 & 9.2 & 41.0 & 11.3 & .488 & 36.5 & 9.0 & 41.5 & 13.1 & .142 \\
\hline Male & 22 & 95.7 & 19 & 82.6 & .346 & 0 & & 4 & 17.4 & .109 \\
\hline White & 21 & 91.3 & 19 & 82.6 & .665 & 23 & 100.0 & 19 & 82.6 & .10 \\
\hline Married & 20 & 87.0 & 19 & 82.6 & $>.999$ & 20 & 87.0 & 19 & 82.6 & $>.999$ \\
\hline Years married $(M$ and $S D)$ & 7.0 & 8.0 & 14.3 & 18.9 & .098 & 6.9 & 7.9 & 11.5 & 11.7 & .124 \\
\hline Veteran & 23 & 100.0 & 23 & 100.0 & - & 1 & 4.3 & 3 & 13.0 & - \\
\hline Employed & 12 & 52.2 & 14 & 60.9 & .552 & 18 & 78.3 & 12 & 52.2 & .063 \\
\hline Education, year ( $M$ and $S D$ ) & 14.3 & 1.8 & 14.7 & 1.6 & .500 & 14.3 & 1.8 & 14.3 & 1.8 & $>.999$ \\
\hline \multicolumn{11}{|l|}{ Index trauma } \\
\hline Combat & 22 & 95.7 & 21 & 91.3 & .550 & - & - & - & - & - \\
\hline TBI & 13 & 56.5 & 3 & 13.0 & $.002^{*}$ & 2 & 13.3 & 0 & 0 & .212 \\
\hline \multicolumn{11}{|l|}{ Reported diagnoses } \\
\hline Anxiety & 4 & 17.4 & 4 & 17.4 & $>.999$ & 1 & 4.3 & 4 & 17.4 & .346 \\
\hline Depression & 4 & 17.4 & 6 & 26.1 & .475 & 8 & 34.8 & 7 & 30.4 & .753 \\
\hline Other & 4 & 17.4 & 4 & 17.4 & $>.999$ & 0 & 0 & 2 & 8.7 & .489 \\
\hline \multicolumn{11}{|l|}{ Stable psychotropic } \\
\hline medications & 17 & 73.9 & 21 & 91.3 & .243 & 7 & 30.4 & 11 & 47.8 & .227 \\
\hline Concurrent psychotherapy & 5 & 21.7 & 3 & 13.0 & .700 & 1 & 4.4 & 5 & 21.7 & .187 \\
\hline \multicolumn{11}{|l|}{ Outcomes $(M$ and $S D)$} \\
\hline CAPS & 71.1 & 12.4 & 70.4 & 13.9 & .859 & - & - & - & - & \\
\hline PCL & 57.6 & 7.8 & 57.2 & 10.5 & .887 & 50.2 & 15.5 & 51.5 & 10.8 & .742 \\
\hline DAS & 99.7 & 19.2 & 96.0 & 25.0 & .577 & 107.6 & 17.2 & 95.7 & 21.9 & $.046^{*}$ \\
\hline BDI-II & 25.6 & 9.5 & 26.8 & 9.6 & .669 & 11.6 & 9.1 & 13.0 & 9.2 & .614 \\
\hline STAI-S & 51.9 & 8.3 & 49.8 & 12.7 & .513 & 34.3 & 12.3 & 38.7 & 12.7 & .248 \\
\hline \multicolumn{11}{|l|}{ STAXI-II subscales } \\
\hline Trait & 22.5 & 5.0 & 25.1 & 6.0 & .116 & 17.5 & 6.7 & 16.9 & 6.3 & .770 \\
\hline Anger expression & 50.8 & 10.2 & 50.1 & 12.2 & .835 & 30.1 & 16.4 & 33.6 & 13.7 & .444 \\
\hline
\end{tabular}

Note. $\quad$ STAXI-II $=$ State-Trait Anger Expression Inventory-II $;$ CAPS $=$ Clinician-Administered PTSD Scale $;$ PCL $=$ PTSD Checklist; BDI-II = Beck Depression Inventory-2nd edition; DAS = Dyadic Adjustment Scale; STAI = State Trait Anxiety Inventory; PTSD = posttraumatic stress disorder; MB-CBCT = mindfulness-based cognitive behavioral conjoint therapy; CBCT-CS = cognitive behavioral conjoint therapy-communication skills.

${ }^{\text {a }}$ Significantly different at $p<0.05$.

teacher certification and attended two silent retreats. All study therapists and doctoral-level trainees participated in an MBSR course and attended weekly mindfulness practice sessions led by the first or second author. Study therapists also received weekly group supervision and individual consultation with the first author for MB-CBCT. Doctoral-level trainees presented segments of the intervention and/or assisted couples with in-session practice during the retreat and postretreat sessions. They were trained and supervised by the first and second authors. Treatment fidelity for MB-CBCT was monitored during each retreat session by trained staff and reported to the interventionists after each retreat session. Interventionists then included any element that was either left out or not covered fully during the next retreat session. MB-CBCT postretreat couple sessions were videotaped and $10 \%$ were chosen at random for review by a CBCT and MB-CBCT trained doctoral student. Fidelity ratings were reviewed by the study interventionists during weekly supervision. Adherence was excellent, with $94.8 \%$ of the essential elements of the therapy delivered (e.g., psychoeducation, in-session skills practice). The mean competency rating was 5.7 on a scale of $1-7(5=\operatorname{good}$ and $6=$ very good).

\section{Comparison Group-CBCT-CS}

To date, no published CBCT studies have compared CBCT to an active control. Since MB-CBCT had not yet been evaluated, it seemed 
premature to compare it to another PTSD-specific intervention such as prolonged exposure or cognitive processing therapy. In order to optimize recruitment and retention in this pilot study, we deemed it of primary importance that the control group be desirable and credible, providing content of interest to postdeployment couples and the retreat format similar to MB-CBCT. Thus, for the control group, we adapted CBCT Phase 1 and Phase 2 to be conducted during a weekend retreat, followed by two monthly multicouple review sessions. The resulting adaptation (CBCT-CS) focused on communication skills and did not include any PTSD-specific education or interventions other than noting the link in the research between PTSD and relationship functioning in order to provide rationale for CBCT-CS. Given that there is less content in CBCT-CS compared with MB-CBCT, there were significantly fewer therapy hours in CBCT-CS. For example, MBCBCT participants attended $13.5 \mathrm{hr}$ of therapy postretreat compared to $3 \mathrm{hr}$ for CBCT-CS participants. However, we reasoned that this disparity in therapy time/attention was less significant than the waitlist or treatment as usual control conditions that are more typical for pilot studies.

The comparison group, CBCT-CS (Davis et al., 2014), includes the communication skills training from Sessions 1-7 of the CBCT for PTSD protocol and was delivered during a weekend retreat followed by two monthly $90 \mathrm{~min}$ multicouple group therapy sessions during which skills use at home was discussed and fine-tuned. CBCT-CS does not include any PTSD-specific or mindfulness interventions. More specifically, at the beginning of the retreat, therapists briefly provided rationale for CBCT-CS by mentioning the research-documented link between PTSD and relationship functioning as well as the link between thoughts, feelings, and behaviors pertaining to the relationship. CBCT-CS did not include CBCT PTSD-specific interventions such as PTSD psychoeducation, PTSD-targeted goal setting, Trauma Impact Questions, trauma disclosure, trauma references in communication skills practice prompts, in-vivo exposure assignments or cognitive interventions. The CBCT-CS therapist was a doctoral-level clinician who was not one of the MB-CBCT therapists but was experienced in delivering communication skills training and manualized treatments in a group setting. She was trained by the first author who provided consultation as needed. Doctoral-level trainees and a bachelor's-level research assistant presented some segments of the CBCT-CS intervention under supervision and assisted couples with in-session practice. Treatment fidelity for CBCT-CS was monitored during each retreat session by trained staff and reported to the interventionists after each retreat session. Interventionists then included any element that was either left out or not covered fully during the next retreat session. Fidelity was not evaluated for the two CBCT-CS postretreat sessions since these sessions did not involve delivering new content but rather provided troubleshooting and review of skills learned during the retreat based on the needs of couples attending the sessions.

\section{Measures}

Study measures chosen are all considered the "gold standard" for the outcome being evaluated and demonstrate very good validity and reliability. Internal consistency (Cronbach's alpha) was estimated for all self-reported measures and was very high for most scales based on the standardized Cronbach's alpha (range 0.90-0.95). Internal consistency was 0.66 for the CAPS, 0.88 for the PCL, and 0.77 for the STAXI-II Anger Expression Subscale. See Supplemental Materials Document 1 for correlations between outcome measures.

The Clinician-Administered PTSD Scale (CAPS; Blake et al., 1995) is a semistructured interview that evaluates PTSD symptoms and diagnostic status according to the Diagnostic and Statistical Manual of Mental Disorders (4th ed., text rev.; DSM-IV-TR; APA, 2000). The intensity and frequency of each PTSD symptom are rated separately on a 5-point Likert scale ranging from 0 to 4 , such that higher scores represent greater symptom severity. Total scores for each symptom cluster (reexperiencing, avoidance and numbing, and hyperarousal) are summed to provide a total symptom severity score ranging from 0 to 136 . For the purposes of this study, a total CAPS severity score of 45 or greater and the required number of symptoms for each cluster that met a minimum frequency of " 1 " and a minimum intensity of " 2 ," confirmed a diagnosis of PTSD. All CAPS raters were doctoral-level clinicians or trainees and blinded to treatment condition. An expert from the National Center for PTSD provided initial CAPS training and provided phone consultation as needed for the duration of the study. CAPS 
interviews were videotaped, and all CAPS assessors rated one interview in common bimonthly during the study. Meetings were held to discuss divergent ratings and establish a consensus rating. The intraclass correlation among study CAPS raters was excellent ( 0.99 for CAPS total score).

The PTSD Checklist (PCL; Weathers et al., 1993) is a 17-item self-report checklist based on the DSM-IV-TR diagnostic criteria for PTSD and was completed separately by Veterans and their partners to indicate their perceptions of the Veteran's PTSD symptoms. Each item is rated on a 5-point Likert scale $(1=$ not at all, $5=$ extremely $)$ and scores range from 17 to 85 , with higher scores indicating greater PTSD symptom severity. In addition to the three subscales scores that represent each symptom cluster (reexperiencing, avoidance/ numbing, and hypervigilance), a total score represents overall symptom severity.

The Dyadic Adjustment Scale (DAS; Spanier, 1976) is a 32-item self-report inventory designed to measure each partner's perception of the quality of an intimate dyadic relationship. The DAS produces a total score (range 0-151) as well as four subscale scores: Dyadic Consensus, Dyadic Satisfaction, Dyadic Cohesion, and Affectional Expression. A total score of 98 or higher is in the satisfied range.

The Conflict Tactics Scale-Revised (CTS-R; Straus, et al., 1996) is a self-report measure that was completed by both Veterans and partners to evaluate the presence of severe aggression. Couples were excluded if they endorsed severe physical or sexual aggression in the past year such as hitting or threatening with a weapon.

Veterans and partners completed four additional measures. The Beck Depression Inventory-2nd edition (BDI-II; Beck, Steer, \& Brown., 1996) is a 21-item self-report measure of depressive symptoms. A total score is calculated by summing the values (0-3) of the endorsed statements (range 0-63), with higher scores indicating more depressive symptoms. This study used the State-Trait Anxiety Inventory (STAI; Spielberger, 1983) 20-item state anxiety subscale (range 20-80). A total score represents the sum of values endorsed on a 4-point Likert scale $(0=$ not at all; $3=$ very much so) with higher scores indicating greater state anxiety. The State-Trait Anger Expression Inventory-II (STAXI-II; Spielberger, 1999) is a 57-item self-report measure used to assess anger expression, experience, and control. This study used the trait subscale (range 10-40) that measures how anger is experienced over time and the anger expression subscale (range 0-72). Item responses range $0-3$ and are summed to yield a total score. Higher scores indicate greater levels of anger. The Client Satisfaction Questionnaire (CSQ-8; Larsen et al., 1979) is an 8-item self-report measure of satisfaction with health and human services that is rated on a 1-4 Likert scale. This measure was collected at treatment end. Item responses are summed to yield a total score which ranges from 8 to 32 . Higher scores indicate higher satisfaction.

\section{Data Analysis}

In accordance with intention-to-treat procedures, all participants were encouraged to complete assessments regardless of treatment completion. This study planned to enroll 48 couples randomized to MB-CBCT or CBCT-CS. Assuming that approximately 40 couples would remain following the retreat and that an additional $10 \%$ would dropout by treatment end, this would provide 18 evaluable Veterans per group by treatment end. This study was powered to detect meaningful differences between treatment group CAPS scores at treatment end. Based on pilot data (Monson et al., 2009), we expected a 1.47 standard deviation $(S D)$ difference in mean CAPS in the MB-CBCT group, assuming no more than a $.5 S D$ difference in mean CAPS in the CBCT-CS group. Thus, 18 subjects per treatment group provide $80 \%$ power to detect this effect size at treatment end based on a two-sample $t$ test with type I error set at $p=.05$. This provides similar power compared to results obtained from our linear mixed model.

Demographic and behavioral characteristics were compared by treatment group using appropriate tests (two-sample $t$ test, chi square, or Fisher's exact test). For each outcome, a linear mixed model was fit to all time points for Veterans and partners separately. This model included fixed effects for treatment, time, and treatment $\times$ time interaction, with time as a categorical variable. An unstructured covariance matrix for the repeated measures was assumed. Adjusted means and contrasts of interest were obtained from the models to test the primary hypotheses that MB-CBCT would result in greater improvement in PTSD symptoms and relationship satisfaction for Veterans and their partners than CBCT-CS at treatment end. Within-group and between-group Hedge's $g$ effect sizes with a correction for sample size (Cooper \& Hedges, 1994; Cumming, 2012) were estimated based on the 
adjusted means from the model results and the observed standard deviation as in the previous CBCT for PTSD study (Monson et al., 2004). A commonly used interpretation for $g$ is to refer to effect sizes as large $(g \geq 0.80)$, medium $(0.50 \leq g \leq 0.79)$, and small $(0.20 \leq g \leq 0.49)$ with negative values indicating a worsening of the symptom being measured (Hedges \& Olkin, 1985). The mixed model approach allows all the data available at each time point to be used assuming that missing data are missing-at-random (MAR). To determine if there were any systematic differences between those who completed treatment end assessments and those who did not, primary and secondary outcomes as well as demographic variables at baseline were compared separately for Veterans and partners in both conditions using a two-sample $t$ test, chi square, or Fisher's exact test. Sensitivity analyses included reestimating the models while controlling for variables that significantly differed at baseline, as well as using multiple imputation to examine the robustness of results under varying scenarios if the data were missingnot-at-random. All data analyses were performed using SAS software, version 9.4 (Cary, NC).

\section{Results}

Our primary hypothesis, that MB-CBCT would lead to greater improvement at treatment end in the primary outcomes of Veterans' PTSD symptoms (CAPS) and Veterans' and partners' relationship satisfaction (DAS) compared to CBCT-CS, was not confirmed. See Table 2 for linear mixed model results. Between-group effect sizes of primary outcomes and PCL scores at end of treatment are provided in Table 3 for both Veterans and partners.

We also predicted that MB-CBCT would result in greater improvement at treatment end in secondary outcomes, including Veteran and partner reports of Veterans' PTSD symptoms, as well as Veterans' and partners' anxiety, depression, and anger. Results for all secondary outcomes except partner-reported PCL scores are included in Supplemental Table 1. For Veterans, there were no statistically significant differences between MBCBCT and CBCT-CS on any of the secondary outcomes at treatment end with the exception of partner-rated PCL scores. MB-CBCT partner-rated PCL scores at treatment end yielded significantly larger improvements compared with CBCT-CS. We found no significant differences between
MB-CBCT and CBCT-CS for primary or secondary outcomes at 6-month follow-up. Regarding the Client Satisfaction Questionnaire, MB-CBCT Veterans indicated significantly greater satisfaction with treatment at treatment end than CBCT-CS Veterans $(M=28.8, S D=2.9 ; M=25.5, S D=$ 4.9, respectively; two-sample $t$ test $p=.026$ ). Similarly, MB-CBCT partners endorsed higher satisfaction than CBCT-CS partners $(M=30.3, S D=2.1 ; M=27.9, S D=3.1$, respectively; two-sample $t$ test $p=.022$ ).

Regarding within-group differences, for Veterans, the change from baseline was statistically significant for all outcomes regardless of treatment group. For partners, both MB-CBCT and CBCT$\mathrm{CS}$ resulted in a significant reduction in depression (BDI-II) and an increase in relationship satisfaction (DAS) at treatment end. However, changes were not statistically significant for either of the STAXI-II subscales measuring anger for partners. The partner-rated PCL scores were only significantly reduced within MB-CBCT, whereas STAI state anxiety subscale scores were significantly reduced only within CBCT-CS. As depicted in Table 3 and Supplemental Table 1, within-group changes at treatment end for both the MB-CBCT and CBCT-CS Veterans and partners improved in the direction expected for all outcomes.

We performed two secondary analyses. First, for all outcomes (except DAS), sensitivity analyses were conducted (see Supplemental Table 2). Contrasts of interest were estimated from linear mixed models that included history of Traumatic Brain Injury (TBI) and baseline DAS since these were the only patient characteristics that significantly differed between treatment groups at baseline. MB-CBCT Veterans were more likely to have endorsed TBI than CBCT-CS Veterans and MB-CBCT partners had a lower mean DAS at baseline than CBCT-CS partners. Results for primary outcomes were similar to the original results. For the outcome of DAS, we also examined this linear mixed model adjusting for TBI but results were not significantly different. Therefore, original results unadjusted for TBI and DAS are reported. Second, we examined differences in demographic variables, primary outcomes, and secondary outcomes measured at baseline between subjects that completed and did not complete treatment end assessments within any of the groups (Veterans in MB-CBCT, Veterans in CBCT-CS, partners in MB-CBCT, and partners in CBCT-CS). The only differences found were within Veterans in CBCT-CS. Veterans in the CBCT-CS group who 
Table 2

Estimates of Fixed Effects for Primary Outcomes

\begin{tabular}{|c|c|c|c|c|c|c|}
\hline Outcome & Effect & $b$ & $S E$ & $d f$ & $t$ & $p$ value \\
\hline \multirow[t]{15}{*}{ CAPS } & Intercept & 47.39 & 5.28 & 44 & 8.98 & $<.001$ \\
\hline & Group & & & & & \\
\hline & CBCT-CS & -8.54 & 8.33 & 44 & -1.03 & .311 \\
\hline & MB-CBCT & 0 & & & & \\
\hline & Time & & & & & \\
\hline & Baseline & 23.70 & 5.36 & 44 & 4.43 & $<.001$ \\
\hline & Treatment end & -1.86 & 3.65 & 44 & -0.51 & .613 \\
\hline & 6 month follow-up & .0 & & & & \\
\hline & Group ${ }^{*}$ Time & & & & & \\
\hline & CBCT-CS, baseline & 7.85 & 8.43 & 44 & 0.93 & .357 \\
\hline & CBCT-CS, Tx end & 9.24 & 5.93 & 44 & 1.56 & .126 \\
\hline & CBCT-CS, 6 months & 0 & & & & \\
\hline & MB-CBCT, baseline & 0 & & & & \\
\hline & MB-CBCT, Tx end & 0 & & & & \\
\hline & MB-CBCT, 6 months & 0 & & & & \\
\hline \multirow[t]{18}{*}{ DAS-Veteran } & Intercept & 102.83 & 3.65 & 44 & 28.14 & $<.001$ \\
\hline & Group & & & & & \\
\hline & CBCT-CS & 2.02 & 5.31 & 44 & 0.38 & .706 \\
\hline & MB-CBCT & 0 & & & & \\
\hline & Time & & & & & \\
\hline & Baseline & -3.14 & 3.27 & 44 & -0.96 & .343 \\
\hline & Treatment end & 11.38 & 2.91 & 44 & 3.91 & $<.001$ \\
\hline & 6-month follow-up & 9.81 & 2.86 & 44 & 3.43 & .001 \\
\hline & Midtreatment & 0 & & & & \\
\hline & Group ${ }^{*}$ Time & & & & & \\
\hline & CBCT-CS, baseline & -5.71 & 4.78 & 44 & -1.19 & .24 \\
\hline & CBCT-CS, Tx end & -4.73 & 4.62 & 44 & -1.02 & .31 \\
\hline & CBCT-CS, 6 months & -7.47 & 4.61 & 44 & -1.62 & .11 \\
\hline & CBCT-CS, Mid Tx & 0 & & & & \\
\hline & MB-CBCT, baseline & 0 & & & & \\
\hline & MB-CBCT, Tx End & 0 & & & & \\
\hline & MB-CBCT, 6 months & 0 & & & & \\
\hline & MB-CBCT, Mid Tx & 0 & & & & \\
\hline \multirow{18}{*}{ DAS-Partner } & Intercept & 107.25 & 3.63 & 44 & 29.54 & $<.001$ \\
\hline & Group & & & & & \\
\hline & CBCT-CS & -6.70 & 5.28 & 44 & $-1,27$ & .211 \\
\hline & MB-CBCT & 0 & & & & \\
\hline & Time & & & & & \\
\hline & Baseline & 0.36 & 2.28 & 44 & 0.16 & .876 \\
\hline & Treatment end & 7.82 & 1.89 & 44 & 4.14 & $<.001$ \\
\hline & 6 month follow-up & 3.35 & 4.39 & 44 & 0.76 & .449 \\
\hline & Midtreatment & 0 & & & & \\
\hline & Group ${ }^{*}$ Time & & & & & \\
\hline & CBCT-CS, baseline & -5.21 & 3.45 & 44 & -1.51 & .13 \\
\hline & CBCT-CS, Tx end & -4.85 & 3.21 & 44 & -1.51 & .14 \\
\hline & CBCT-CS, 6 months & -5.42 & 7.36 & 44 & -0.74 & .47 \\
\hline & CBCT-CS, Mid Tx & 0 & & & & \\
\hline & MB-CBCT, baseline & 0 & & & & \\
\hline & MB-CBCT, Tx end & 0 & & & & \\
\hline & MB-CBCT, 6 months & 0 & & & & \\
\hline & MB-CBCT, Mid Tx & 0 & & & & \\
\hline
\end{tabular}

Note. $\mathrm{MB}-\mathrm{CBCT}=$ mindfulness-based cogntive behavioral conjoint therapy; CBCT-CS $=$ cognitive behavioral conjoint therapy-communication skills.

did not complete the treatment end assessments $(N=10)$ were less educated $(p=.025)$ and had higher PCL total scores $(p=.033)$ than those who did complete the assessments $(N=13)$. Thus, additional sensitivity analyses were conducted under missing-not-at-random scenarios. However, results indicate there was no significant difference in change in CAPS score at treatment end between groups. 
DAVIS ET AL.

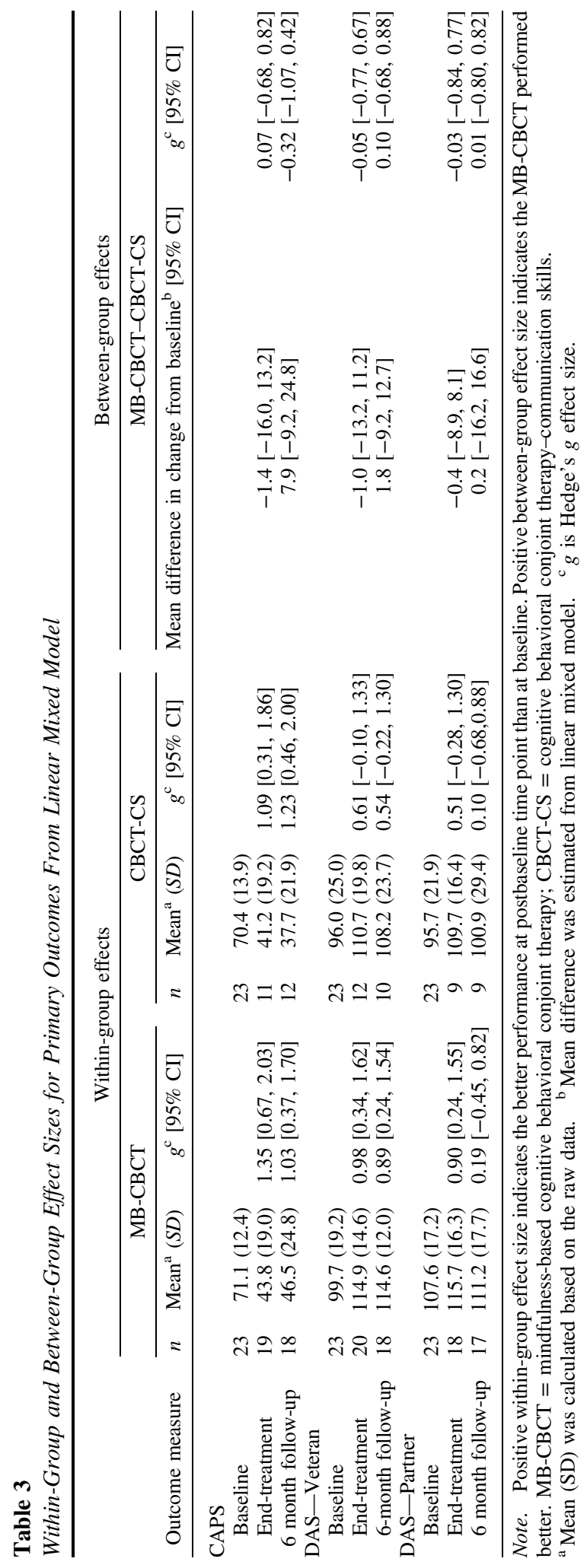




\section{Discussion}

This randomized controlled pilot study with 46 couples compared the effects of MB-CBCT, a 10 week mindfulness-based adaptation of CBCT for PTSD, with CBCT-CS, a 10 week version of CBCT offering only communication skills training, on PTSD symptoms and relationship satisfaction. Although there was no significant difference between groups at treatment end, Veterans in both treatment groups demonstrated improved CAPS rated PTSD symptoms, with large within-group effect sizes. MB-CBCT and CBCT-CS Veterans and their partners also significantly improved on relationship satisfaction as measured by the Dyadic Adjustment Scale (MB-CBCT Veterans and partners, large within-group effect sizes; CBCT-CS Veterans and partners, medium effect sizes).

There are several possible explanations for the lack of significant difference between MB-CBCT and CBCT-CS. First, perhaps the briefer, communication-focused CBCT-CS was sufficient to improve relationship satisfaction and thereby reduce PTSD symptoms for CBCT-CS couples who completed treatment. Many studies demonstrate positive effects of communication skills training on relationship quality (see Stanley et al., 2020). PTSD symptom reduction is associated with treatments that include a communications skills component, for example, dialectical behavior therapy along with weekly writing assignments about positive and negative experiences (Bradley \& Follingstad, 2003), STAIR narrative therapy without exposure (Landes et al., 2013) and structured approach therapy, a conjoint PTSD treatment (Sautter et. al., 2015). Thus, this is the only study to our knowledge that evaluates the effects of communications skills alone on PTSD symptoms.

Second, CBCT-CS partners were in the distressed range on the baseline DAS whereas the MB-CBCT partners were in the satisfied range, which may have been a factor in the stronger than expected results for CBCT-CS couples. In fact, Shnaider et al. (2015) reported greater improvements in relationship satisfaction for partners in the distressed range in a follow-up analysis of a randomized clinical trial of CBCT (Monson et al., 2012). Third, the unexpected high dropout from CBCT-CS (43\% vs. MB-CBCT, 13\%) may have limited our ability to detect statistically significant differences between MB-CBCT and
CBCT-CS. Results of the CSQ-8, which measures treatment satisfaction, revealed that both MB-CBCT Veterans and partners regarded the MB-CBCT program more favorably than the CBCT-CS Veterans and partners which could have contributed to higher CBCT-CS dropout. Perhaps childcare compensation, such as provided by Fredman et al. (2019) in their study, may have improved retreat attendance in the CBCT-CS group (78\% vs. $96 \%$ in MB-CBCT). Of note, Veterans in the CBCT-CS group who did not complete treatment end assessments were less educated and had higher baseline PCL scores than those who did complete them. Even though the sensitivity analyses we conducted under missingnot-at-random scenarios indicated there was no significant difference in change in CAPS at treatment end between groups, we cannot rule out the possibility that dropout was a factor that could have contributed to the lack of between-group differences.

Finally, although there was a large effect size change in CAPS for MB-CBCT Veterans, it was smaller than the effect sizes seen in Monson and colleagues' study (2012). However, the CAPS effects size disparity between studies may be partially explained by several differences between the studies. First, Monson and Fredman's (2012) study sample included more female participants with PTSD (65\% vs. $11 \%)$. One meta-analysis reported females experience better outcomes than males in PTSD treatment (Watts et al., 2013). Second, the CBCT study sample included more non-Veteran participants with PTSD $(22.5 \%$ vs. $100 \%)$. The same metaanalysis reported that civilians have better outcomes in PTSD treatments than Veterans. Third, CBCT study therapists included the two developers of CBCT. Although treatment fidelity was good in the present study, the therapists had less experience and expertise in delivering the protocol, which may also have impacted the effect sizes favoring the Monson and Fredman's (2012) study. Another possible explanation is that individually delivered interventions for PTSD may produce somewhat stronger outcomes than group-delivered interventions and/or massed group interventions such as the weekend retreats utilized in the present study. In addition, Cougle (2012) suggests more parsimonious treatments may lead to better outcomes, For example, mindfulness content added to CBCT may be too much for some couples. 
The findings of this study must be interpreted cautiously in light of limitations. Our sample size was small and there was disproportionately high dropout in the CBCT-CS group. Our sample included predominantly male Veterans from the Iraq and Afghanistan conflicts, the majority of whom reported a combat-related index trauma, which limits applying these findings to female Veterans and civilians and those whose index trauma is not combat-related. Although we evaluated psychotropic medications and other therapy at baseline and found no differences between groups, we did not monitor these variables throughout the study, which could have impacted findings. We collected information regarding satisfaction with the intervention (CSQ-8) but did not administer a measure of treatment credibility postrandomization which could help make sense of any unexpected findings in future studies.

Nonetheless, this study has important implications for clinical practice and future research. At the time of this writing, there are two other studies that have investigated variations of CBCT. Pukay-Martin et al. (2015) evaluated seven couples from a community sample who received a present-focused version of CBCT that included in vivo approach assignments for pleasant activities and cognitive work related to present-centered cognitions that were not specific to the trauma but could be related to its effects on couples' lives in the present. This study found statistically significant improvements in both PTSD symptoms and relationship satisfaction. Fredman et al. (2019) conducted a pilot project with 24 couples that included an active duty service member or Veteran who had PTSD to evaluate a briefer version of CBCT delivered to multicouple groups during a weekend retreat. This version included trauma disclosure, in-vivo approach assignments, and cognitive work. Comparison of baseline assessments to those conducted 1 and 3 months postintervention showed medium to large effect sizes for improvements in clinician and patientreported PTSD symptoms and partner-reported PTSD symptoms and relationship satisfaction at 3 months (medium effect sizes). These studies, along with the current study, suggest the promise of offering various forms of conjoint PTSD treatments in clinical settings. Another study currently underway compares a brief 8-session version of CBCT delivered to Veterans and their partners in a traditional VA office setting with the same protocol delivered through videoconferencing and an office-delivered PTSD Family Education control group (Morland et al., 2019). Additional studies are needed to identify which intervention characteristics are essential ingredients for effectiveness, such as content condensed into a briefer time frame (i.e., a weekend retreat) and present focused versus relationship focused versus trauma focused intervention content.

This is the first published randomized controlled pilot study of two adaptations of CBCT delivered to Iraq and Afghanistan Veterans. Findings suggest that $\mathrm{CBCT}$ adaptations (MB-CBCT and CBCT-CS) can be effectively delivered by clinicians other than the treatment developers over fewer total weeks than CBCT by offering Phases 1 and 2 during a weekend retreat. In this small sample, between-group comparisons of primary and secondary outcomes were not statistically significant except for partner-rated PCL. Future research should include larger and more diverse samples to confirm or refute these findings, to determine who might most benefit from these adaptations of CBCT, and to further investigate the role of the mindfulness component and the retreat format in treatment outcome. The findings of this study suggest both MB-CBCT and CBCT-CS may provide efficacious options for efficient delivery of PTSD treatment and choice regarding trauma focus. Study results also point to a need for continued development and evaluation of PTSD treatments that are shorter duration, delivered efficiently, include significant others, and may or may not have a trauma focus.

\section{References}

American Psychiatric Association. (2000). Diagnostic and statistical manual of mental disorders (4th ed., text rev.).

American Psychiatric Association. (2013). Diagnostic and statistical manual of mental disorders (5th ed.). https://doi.org/10.1176/appi.books.9780890425596

Banks, K., Newman, E., \& Saleem, J. (2015). An overview of the research on mindfulness-based interventions for treating symptoms of posttraumatic stress disorder: A systematic review. Journal of Clinical Psychology, 71(10), 935-963.

Beck, A. T., Steer, R. A., \& Brown, G. K. (1996). Manual for the Beck Depression Inventory-II. Psychological Corporation.

Blake, D. D., Weathers, F. W., Nagy, L. M., Kaloupek, D. G., Gusman, F. D., Charney, D. S., \& Keane, T. M. (1995). The development of a clinician 
administered PTSD scale. Journal of Traumatic Stress, 8(1), 75-90. https://doi.org/10.1002/jts .2490080106

Boyd, J. E., Lanius, R. A., \& McKinnon, M. C. (2018). Mindfulness-based treatments for posttraumatic stress disorder: A review of the treatment literature and neurobiological evidence. Journal of Psychiatry \& Neuroscience, 43, 7-25.

Bradley, R. G., \& Follingstad, D. R. (2003). Group therapy for incarcerated women who experienced interpersonal violence: A pilot study. Journal of Traumatic Stress, 16(4), 337-340.

Carson, J. W., Carson, K. M., Gil, K. M., \& Baucom, D. H. (2004). Mindfulness-based relationship enhancement. Behavior Therapy, 35(3), 471-494.

Christensen, A., \& Jacobson, N. S. (2000). Reconcilable differences. The Guilford Press.

Cooper, H., \& Hedges, L. V. (1994). The handbook of research synthesis. Sage Publications.

Cougle, J. R. (2012). What makes a quality therapy? A consideration of parsimony, ease and efficiency. Behavior Therapy, 43, 468-481.

Cumming, G. (2012). Understanding the new statistics: Effect sizes, confidence intervals, and metaanalysis. Routledge/Taylor \& Francis Group.

Davis, L. W. (2016). Mediations for healing trauma: Mindfulness skills to ease post-traumatic stress. New Harbinger Publications.

Davis, L. W., Luedtke, B. L., Monson, C. M. \& Fredman, S. J. (2014). Mindfulness-Based Cognitive-Behavioral Conjoint Therapy. Unpublished treatment manual.

Davis, L. W., Paul, R., Tarr, D., Eicher, A. C., Allinger, J., \& Knock, H. (2012). Operation Restoration: Couples reunification retreats for veterans of operations enduring and Iraqi freedom. Journal of Psychosocial Nursing and Mental Health Services, 50(11), 20-29. https://doi.org/10.3928/0279369520121003-02

Dorrington, S., Zavos, H., Ball, H., McGuffin, P., Sumathipala, A., Siribaddana, S., Rijsdijk, F., Hatch, S. L., \& Hotopf, M. (2018). Family functioning, trauma exposure and PTSD: A cross sectional study. Journal of Affective Disorders, 245 , 645-652. https://doi.org/10.1016/j.jad.2018.11.056

Erbes, C. R., Curry, K. T., \& Leskela, J. (2009). Treatment presentation and adherence of Iraq/ Afghanistan era veterans in outpatient care for posttraumatic stress disorder. Psychological Services, 6(3), 175-183.

Fredman, S. J., MacDonald, A., Monson, C. M., Dondanville, T. H., Blount, B. N., Hall-Clark, B. A., Fina, B. A., Mintz, J., Litz, B. T., YoungMcCaughan, S., Hancock, A. K., Rhoades, G. K., Yarvis, J. S., Resick, P. A., Roache, J. D., Yunying, L., Wachen, J. S., Niles, B. L., McGeary, C. A., . . . Consortium to Alleviate PTSD. (2019). Intensive, multi-couple group therapy for PTSD: A nonrandomized pilot study with military and veteran dyads. Behavior Therapy. 51(5), 700-714. https://doi.org/10.1016/j.beth.2019.10.003

Gambrel, L. E., \& Keeling, M. L. (2010). Relational aspects of mindfulness: Implications for the practice of marriage and family therapy. Contemporary Family Therapy, 32(4), 412-426. https://doi.org/ 10.1007/s10591-010-9129-z

Gottman, J. M. (1993). A theory of marital dissolution and stability. Journal of Family Psychology, 7, 57-75.

Hedges, L. V., \& Olkin, I. (1985). Statistical methods for meta-analysis. Academic Press.

Hoge, C. W. (2011). Interventions for war-related posttraumatic stress disorder: Meeting Veterans where they are. Journal of the American Medical Association, 306(5), 549-551.

Kabat-Zinn, J. (1990). Full catastrophe living: Using the wisdom of your body and mind in everyday life. Dell.

Kearney, D. J., Mcdermott, K., Malte, C., Martinez, M., \& Simpson, T. L. (2013). Effects of participation in a mindfulness program for veterans with posttraumatic stress disorder: A randomized controlled pilot study. Journal of Clinical Psychology, 69, 14-27.

Kimbrough, E., Magyari, T., Langenberg, P., Chesney, M., \& Berman, B. (2010). Mindfulness intervention for child abuse survivors. Journal of Clinical Psychology, 66, 17-33.

King, A. P., Erickson, T. M., Giardino, N. D., Favorite, T., Rauch, S. A. M., Robinson, E., Kulkarni, M., \& Liberzon, I. (2013). A pilot study of group mindfulnessbased cognitive therapy (MBCT) for combat veterans with posttraumatic stress disorder (PTSD). Depression and Anxiety, 30, 638-645.

Landes, S. J., Garovoy, N. D., \& Burkman, K. M. (2013). Treating complex trauma among veterans: Three stage-based treatment models. Journal of Clinical Psychology, 69(5), 523-533.

Larsen, D. L., Attkisson, C. C., Hargreaves, W. A., \& Nguyen, T. D. (1979). Assessment of client/patient satisfaction: Development of a general scale. Evaluation and Program Planning, 2, 197-207.

Liebman, R. E, Whitfield, K. M., Sijerci, I., Ennis, N., \& Monson, C. M. (2020). Harnessing the healing power of relationships in trauma recovery: A systematic review of cognitive behavioral conjoint therapy for PTSD. Current Treatment Options in Psychiatry, 7, 203-220.

Luedtke, B. L., Davis, L., \& Monson, C. (2015). Mindfulness-based cognitive-behavioral conjoint therapy for posttraumatic stress disorder: A case study. Journal of Contemporary Psychotherapy. 45(4), 227-234.

Meis, L. A., Barry, R. A., Kehle, S. M., Erbes, C. R., \& Polusny, M. A. (2010). Relationship adjustment, PTSD symptoms, and treatment utilization among 
coupled National Guard soldiers deployed to Iraq. Journal of Family Psychology, 24, 560-567. https:// doi.org/10.1037/a0020925

Monson, C. M., \& Fredman, S. J. (2012). Cognitivebehavioral conjoint therapy for PTSD: Harnessing the healing power of relationships. The Guilford Press.

Monson, C. M., Fredman, S. J., Macdonald, A., PukayMartin, N. D., Resick, P. A., \& Schnurr, P. P. (2012). Effect of cognitive-behavioral couple therapy for PTSD: A randomized controlled trial. Journal of the American Medical Association, 308(7), 700-709.

Monson, C. M., Fredman, S. J., Stevens, S. P., MacDonald, H. Z., Adair, K. C., Schnurr, P. P., \& Resick, P. A. (2009). Cognitive-behavioral conjoint therapy for PTSD: Pilot study results [Paper presentation]. Anxiety Disorders Association of America Annual Conference, Santa Ana Pueblo, NM, United States.

Monson, C. M., Schnurr, P. P., Stevens, S. P., \& Guthrie, K. A. (2004). Cognitive-behavioral couple's treatment for posttraumatic stress disorder: Initial findings. Journal of Traumatic Stress, 17(4), 341-344. https://doi.org/10.1023/B:JOTS $.0000038483 .69570 .5 b$

Monson, C. M., Shields, N., Suvak, M. K., Lane, J. E. M., Shnaider, P., Landy, M. S. H., Wagner, A. C., Sijercic, I., Masina, T., Wanklyn, S. G., \& Stirman, S. W. (2018). A randomized controlled effectiveness trial of training strategies in cognitive processing therapy for posttraumatic stress disorder: Impact on patient outcomes. Behaviour Research and Therapy, 110, Article 31049. https://doi.org/10 .1016/j.brat.2018.08.007

Morland, L. A., Macdonald, A., Grubbs, K. M., Mackintosh, M. A., Monson, C. M., Glassman, L. H., Becker, J., Sautter, F., Buzzella B., Wrape, E., Wells, S. Y., Rooney, B. M., \& Glynn, S. (2019). Design of a randomized superiority trial of a brief couple treatment for PTSD. Contemporary Clinical Trials Communications, 15, 1-8. https://doi.org/10 $.1016 / j . c o n c t c .2019 .100369$

Pukay-Martin, N. D., Torbit, L., Landy, M. S., Wanklyn, S. G., Shnaider, P., Lane, J. E., \& Monson, C. M. (2015), An uncontrolled trial of a present-focused cognitive behavioral conjoint therapy for posttraumatic stress disorder. Journal of Clinical Psychology, 71, 302-312. https://doi.org/ 10.1002/jclp.22166

Sautter, F. J., Glynn, S. M., Cretu, J. B., Senturk, D., \& Vaught, A. S. (2015). Efficacy of structured approach therapy in reducing PTSD in returning veterans: A randomized clinical trial. Psychological Services, 12(3), 199-212. https://doi.org/10.1037/ser0000032

Shapiro, S. L., Schwartz, G. E., \& Bonner, G. (1998). Effects of mindfulness-based stress reduction on medical and premedical students. Journal of Behavioral Medicine, 21, 581-599.

Shnaider, P., Pukay-Martin, N. D., Sharma, S., Jenzer, T., Fredman, S. J., Macdonald, A., \& Monson, C. M. (2015). A preliminary examination of the effects of pretreatment relationship satisfaction on treatment outcomes in cognitive-behavioral conjoint therapy for PTSD. Couple \& Family Psychology, 4(4), 229-238.

Spanier, G. B. (1976). Measuring dyadic adjustment: New scales for assessing the quality of marriage and similar dyads. Journal of Marriage and the Family, 38(1), 15-28.

Spielberger, C. D. (1983). Manual for the State-Trait Anxiety Inventory (Form Y) (self-evaluation questionnaire). Consulting Psychologists Press.

Spielberger, C. D. (1999). Manual for State-Trait Anger Expression Inventory-2. Psychological Assessment Resources, Inc.

Stanley, S. M., Carlson, R. G., Rhoades, G. K., Markman, H. J., Ritchie, L. L., \& Hawkins, A. J. (2020). Best practices in relationship education focused on intimate relationships. Family Relations: Interdisciplinary Journal of Applied Family Science, 69(3), 497-519.

Straus, M. A., Hamby, S., McCoy, S., \& Sugarman, D. (1996). The Revised Conflict Tactic Scale (CTS2): Development and preliminary psychometric data. Journal of Family Issues, 17(3), 283-316.

Taft, C. T., Watkins, L. E., Stafford, J., Street, A. E., \& Monson, C. M. (2011). Posttraumatic stress disorder and intimate relationship problems: A meta-analysis. Journal of Consulting and Clinical Psychology, 79(1), 22-33. https://doi.org/10.1037/a0022196

Watts, B. V., Schnurr, P. P., Mayo, L., Young-Xu, Y., Weeks, W. B., \& Friedman, M. J. (2013). Meta-analysis of the efficacy of treatments for posttraumatic stress disorder. The Journal of Clinical Psychiatry, 74(6), 551-557.

Weathers, F. W., Litz, B. T., Herman, J. A., Huska, J. A., \& Keane, T. M. (1993, November). The PTSD Checklist (PCL): Reliability, validity and diagnostic utility [Paper presentation]. Paper presented at the 9th annual conference of the International Society for Traumatic Stress Studies, San Antonio, TX, United States.

Wenzel, A., \& Harvey, J. (2001). Introduction: The movement toward studying the maintenance and enhancement of close romantic relationships. In J. Harvey \& A. Wenzel (Eds.), Close romantic relationships: Maintenance and enhancement (pp. 1-10). Erlbaum.

Received May 15, 2020

Revision received November 22, 2020

Accepted February 8, 2021 